\title{
System-reliability analysis of steel truss towers
}

\author{
Wojciech Mochocki ${ }^{1,}{ }^{*}$, Paulina Obara ${ }^{1}$, and Urszula Radon $^{1}$ \\ ${ }^{1}$ Kielce University of Technology, Faculty of Civil Engineering and Architecture, \\ al. Tysiąclecia PP 7, 25-314 Kielce, Poland
}

\begin{abstract}
Steel truss towers are important structures in civil engineering, used as, for example, antenna towers, architectural towers, transmission towers, chimney towers, light towers, viewing towers, or water towers. In the paper, the latter structural type is analysed. Three types of truss towers with different topology are discussed. The towers are designed according to the standards. Next, reliability analysis is performed and the influence of type of truss topology on reliability is analysed. Reliability analysis concerns formulation of the limit state, calculation of failure probability and determination of reliability index. In the paper, the system approach is proposed, definitely more complete than that obtained using approximation-simulation methods. In the case of structures analysed in the paper, the types of reliability models are determined. Identification of reliability models relies on studies on the transformation of the safe structural system into the geometrically variable system (mechanism). The investigations made it possible to determine the kinematically admissible failure mechanisms which contain minimal critical sets of elements. In order to identify the mechanism, spectral analysis of the linear stiffness matrix is used. In the case of the towers of concern, the formulas specifying the number of mechanisms for any number of repeating sections are determined.
\end{abstract}

\section{Introduction}

Towers are stand-alone structures rigidly fixed to the base. A number of tower types can be distinguished due to their use [1]. Antenna towers are tall structures designed to support antennas for telecommunications and broadcasting, architectural towers commemorate historic events, transmission towers (electricity pylons) support power lines, chimney towers provide support for flue systems, light towers offer the possibility of installing powerful lighting, viewing towers have observation decks providing extensive views, and finally water towers are used to pressurize a water supply system for water distribution.

In many cases, steel truss tower is considered the most effective structural option. Such a solution makes it possible to build to greater heights than the case of reinforced concrete or masonry structures. Truss towers use lower amounts of material than solid structures, and additionally, they are easy to construct and assemble. However, as in the case of pinjointed multi-element steel structures, reliability assessment is an issue of key

\footnotetext{
* Corresponding author: wojtek1391@gmail.com
} 
importance [2]. The structure is reliable if it performs functions specified in the design over the entire service life. A usual measure of reliability is the reliability index $\beta$ [3].

The methods commonly used for the structural reliability assessment can be classified as follows [4]: Level I, Level II, Level III methods and the system approach. Level I, i.e. semi-probabilistic methods involve the verification of structural reliability, but they do not determine the exact failure probability. Those methods use partial safety factors. Level II methods include approximation (FORM, SORM) and simulation (Monte Carlo, Importance Sampling) methods. In Level II methods, boundary probability distributions of individual random variables are used $[3,4]$. Level III methods are fully probabilistic and they provide an extension of Level II methods. In this case, the cumulative probability distribution of random variables is known. Another group contains system approach (serial, parallel and mixed systems). In this case structural characteristics of the building must be taken into account, i.e. interaction of the structural elements in the load-carrying system [3, 5-8]. In the paper, the system approach is used to assess the reliability.

\section{Truss tower design}

In the paper, reliability analysis is conducted for towers exhibiting different truss types. Water tower with a water tank located at the top of the structure is designed in accordance with current standards [2, 9-11]. The structural model is shown in Fig.1, the truss types can be seen in Fig. 2.

According to the design, the tower is situated in the wind speed zone I, in the roughness class 2. The structure foundation is at $A=170 \mathrm{~m}$ a.s.l. The wind load is modelled in accordance with the loading scheme appropriate for this structure [11, 12]. The main permanent load is a cylindrical steel sheet water tank that is filled with water. Additionally, the dead load, the weight of the $3 \times 3 \mathrm{~m}$ steel deck, on which the tank is installed, and the deck service load of $2 \mathrm{kN} / \mathrm{m}^{2}$ are taken into account.

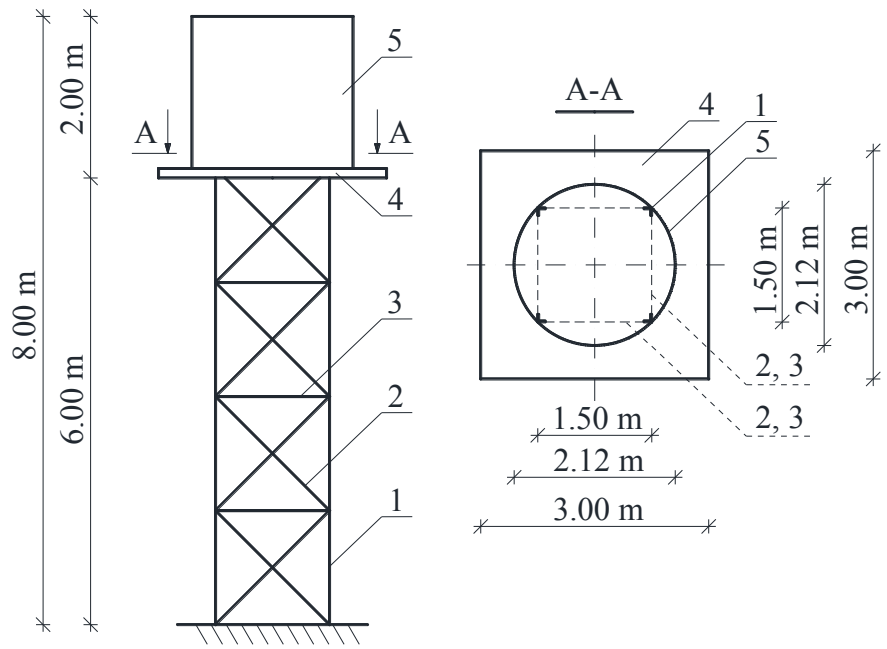

Fig. 1. Scheme of the tower with a tank (1 - main legs, 2 - diagonal bracing, 3 - horizontal bracing, 4 - deck, 5 - water tank).

In the paper three cases of truss topology namely W1, W2, and W3 are discussed. In Fig. 2 truss topologies for $n=4$ repeatable sections are presented. Table 1 shows steel sections, numbers of the most stressed elements and their stress intensity level depending 
on the assumed truss type. The towers are made of S235 steel of the yield strength $f_{y}=235$ $\mathrm{MPa}$.

a)

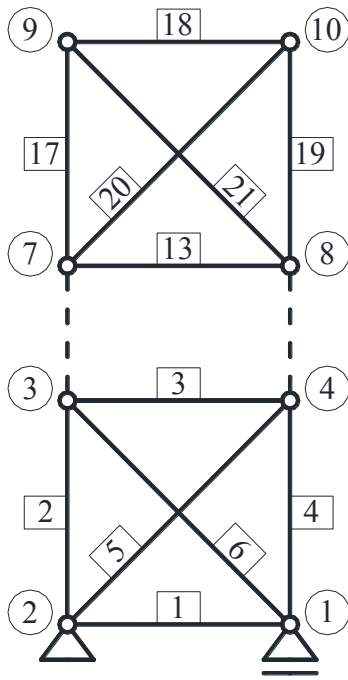

b)

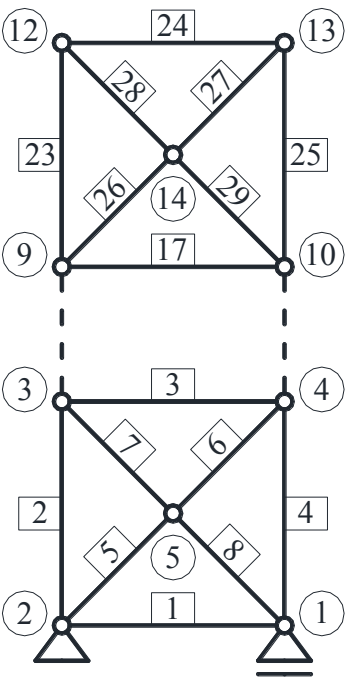

c)

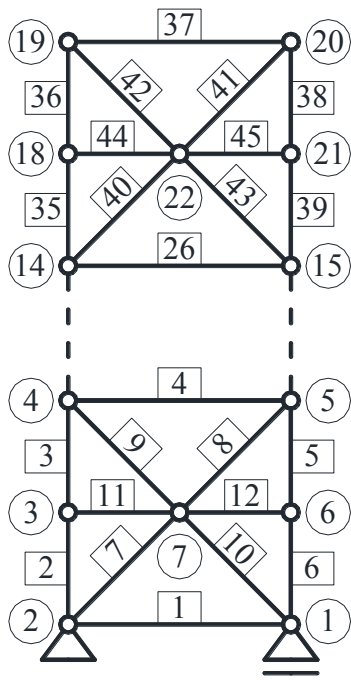

Fig. 2. Truss topology: a) W1, b) W2, c) W3.

Table 1. Structural elements of truss towers.

\begin{tabular}{|c|c|c|c|c|}
\hline \multirow{2}{*}{$\begin{array}{c}\text { Truss } \\
\text { topology }\end{array}$} & \multirow{2}{*}{$\begin{array}{c}\text { Element } \\
\text { profile }\end{array}$} & \multicolumn{2}{|c|}{ Most stressed element } \\
\cline { 4 - 5 } & & Element type & & $\begin{array}{c}\text { Stress intensity level } \\
{[\%]}\end{array}$ \\
\hline \multirow{3}{*}{ W1 } & main leg & L50x6 & 2 & 78 \\
\cline { 2 - 5 } & diagonal bracings & L40x5 & 5 & 73 \\
\cline { 2 - 5 } & horizontal bracings & L40x4 & 3 & 11 \\
\hline \multirow{3}{*}{ W2 } & main leg & L50x6 & 2 & 78 \\
\cline { 2 - 5 } & diagonal bracings & L40x5 & 5 & 73 \\
\cline { 2 - 5 } & horizontal bracings & L40x4 & 3 & 11 \\
\hline \multirow{3}{*}{ W3 } & main leg & L40x4 & 2 & 65 \\
\cline { 2 - 5 } & diagonal bracings & L45x6 & 7 & 81 \\
\cline { 2 - 5 } & horizontal bracings & L40x4 & 4 & 20 \\
\hline
\end{tabular}

\section{Reliability analysis of the truss}

\subsection{System-reliability}

When the system approach is applied to assess the reliability of a rod structure, it is necessary to consider its structural arrangement and the interaction of structural elements. This way, the reliability model, which is the structure eigen value, can be identified. In order to define the reliability model, the kinematically admissible failure mechanisms (KAFMs) should be specified. They contain the minimal critical sets of elements (MCSE), which have a specified number of causative elements $(l)$. In reliability theory, three system 
types are distinguished, serial, parallel and mixed (parallel-serial and serial-parallel) systems (Fig. 3).

a)

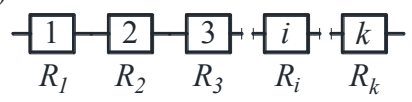

$k \operatorname{MCSE}(l=1)$

d)
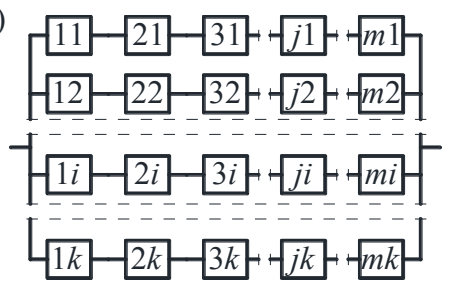

$m k \operatorname{MCSE}(l=k)$ b)

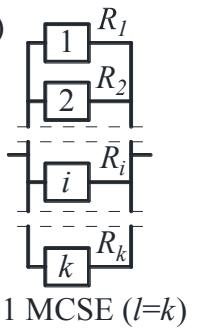

c)

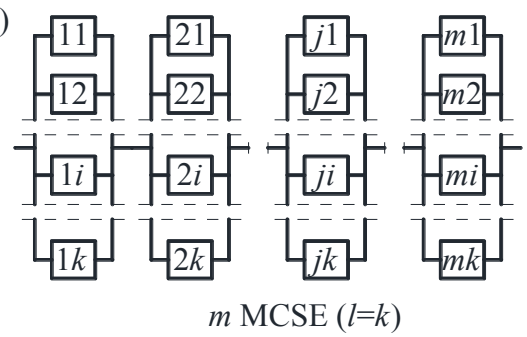

Fig. 3. Types of reliability models: a) serial system, b) parallel system, c) parallel-serial system, d) serial-parallel system.

The serial system (Fig. 3a) is the one which undergoes failure if even only one element fails (becomes inoperable), making the system becomes geometrically variable. The serial system corresponds to statically determinate structures, in which the capacity of the weakest element is decisive for the capacity of the entire structure. The characteristic feature of the serial system is that with the growth of the number of its elements, the capacity and reliability decrease. Reliability of the system $R$ is calculated from the formula:

$$
R=\prod_{i=1}^{k} R_{i}
$$

where:

$R_{i} \quad$ reliability of a single element, $k$ - number of the system elements.

The serial system consists of $k$ minimal critical sets of elements ( $k$ MCSE), each of which has one causative element $(l=1)$.

The parallel system (Fig. 3b) is operable if at least one element is operable. The parallel system corresponds to certain statically indeterminate structures. The capacity and reliability of this system increase with the number of the elements. In this case, one minimal critical set of elements (1 MCSE) is found, which consists of $k$ causative elements $(l=k)$, and the reliability of the system is calculated as follows:

$$
R=1-\prod_{i=1}^{k}\left(1-R_{i}\right)
$$

In the case of mixed (parallel-serial and serial-parallel) systems, in order to identify a proper reliability format of the structure it is necessary to analyse how the elements are coupled, i.e. what relations hold between them. The parallel-serial system (Fig. 3c) consists of $m$ MCSE, each of which includes $k$ causative elements $(l=k)$. The reliability of such a system is computed applying a formula:

$$
R=1-\prod_{j=1}^{m}\left[1-\prod_{i=1}^{k}\left(1-R_{j i}\right)\right]
$$


In contrast, in the case of the serial-parallel system (Fig. 3d), the number of MCSE is $m \cdot k$, and each MCSE consists of $k$ causative elements $(l=k)$. The reliability of such a system is computed acc. formula:

$$
R=1-\prod_{i=1}^{k}\left[1-\prod_{j=1}^{m} R_{j i}\right]
$$

Complex structures may contain systems with different types of connections presented above. That also refers to truss towers, as shown in the paper.

In order to determine reliability of the system, it is also necessary to compute the reliability of a single element. The procedure that leads to the determination of the reliability of element $R_{i}$ was presented, among others, in the paper [7]. In order to determine the element reliability, it is necessary to determine random variables of the effect of action $E_{i}$, and of capacity $N_{i}$. Random variables are described by means of normal distribution with the parameters $\mu$ (expected value) and $\sigma$ (standard deviation). With respect to standard deviation for effect of actions $\sigma_{E i}$, variation coefficient is assumed at the level of $6 \%$, whereas due to standard deviation for capacity $\sigma_{N i}$, its level is $10 \%$. A subsequent step is determining the safety margin $Z_{i}$, which is a difference between the random variable of the effect of action $E_{i}$ and of capacity $N_{i}$. As a result, the safety margin $Z_{i}$ is also a random variable described by means of normal distribution. The parameters of this system are the expected value of the safety margin $\mu_{Z i}=\mu_{N i}-\mu_{E i}$ and standard deviation of the safety margin $\sigma_{Z i}=\sqrt{\sigma_{E i}^{2}+\sigma_{N i}^{2}}$. Next, reliability index for a single element $\beta_{i}=\mu_{Z i} / \sigma_{Z i}$ is computed. On its basis, probability of the element failure can be estimated $P_{f i}=\Phi\left(-t_{i}\right)$, where $\Phi$ is the Laplace function. The final step involves the computation of the element reliability $R_{i}=1-P_{f i}$ and the reliability of the system $R$. In order to compute the reliability of the system, it is necessary to identify the reliability model.

\subsection{Kinematically admissible failure mechanisms for truss towers}

Defining kinematically admissible failure mechanisms (KAFMs) that contain minimal critical sets of elements (MCSE) consists in specifying the possible mechanisms. To that end, spectral analysis of the linear stiffness matrix is performed:

$$
\left(\mathbf{K}_{\mathbf{L}}-\lambda \mathbf{I}\right) \mathbf{q}=\mathbf{0}
$$

where:

$\mathbf{K}_{\mathbf{L}} \quad$ linear stiffness matrix, $\lambda$ - eigen values, $\mathbf{q}$ - displacement vector.

Eigen values $\lambda$ describe the system energy state, whereas the eigen vectors corresponding to eigen values describe the form of deformation. The system is not a mechanism if all eigen values $\lambda$ have a positive value. The mechanism will be found if any of eigen values $\lambda_{i}$ equals zero, and the mechanism form is defined by the eigenvector corresponding to the value $\lambda_{i}[6]$. In order to carry out spectral analysis of the linear stiffness matrix the program developed by the authors in the Mathematica environment is applied.

In the paper, truss towers with three truss topology are analysed (Fig. 2). On the basis of the analyses, formulas are derived for the number of mechanisms $l m$ for each truss (W1, $\mathrm{W} 2$, and $\mathrm{W} 3$ ), depending on the number of elements le and the number of repeating 
sections $n$. With respect to $\mathrm{W} 1$ and $\mathrm{W} 2$ topologies, the number of mechanisms is given by the formula:

$$
\operatorname{lm}=\frac{(l e-(n-1)) !}{2 !((l e-(n-1))-2) !}
$$

whereas for W3 topology:

$$
l m=\frac{(l e-(n-1)-2 n) !}{2 !((l e-(n-1)-2 n)-2) !}+2 n
$$

For instance, Table 2 shows the number of mechanisms $l m$ depending on truss topology, for the number of repeating sections from 1 to 4 .

Table 2. Number of mechanisms.

\begin{tabular}{|c|c|c|c|c|c|}
\hline \multirow{2}{*}{ Truss topology } & \multirow{2}{*}{$\begin{array}{c}\text { Formula for the } \\
\text { number of tower } \\
\end{array}$} & \multicolumn{4}{|c|}{ Number of repeating sections $n$} \\
\cline { 3 - 6 } & elements $l e$ & 1 & 2 & 3 & 4 \\
\cline { 3 - 6 } & $6+5(n-1)$ & 15 & 45 & 91 & 153 \\
\hline W1 & $8+7(n-1)$ & 28 & 91 & 190 & 325 \\
\hline W2 & $12+11(n-1)$ & 47 & 157 & 331 & 569 \\
\hline W3 & & \multicolumn{4}{|c}{} \\
\hline
\end{tabular}
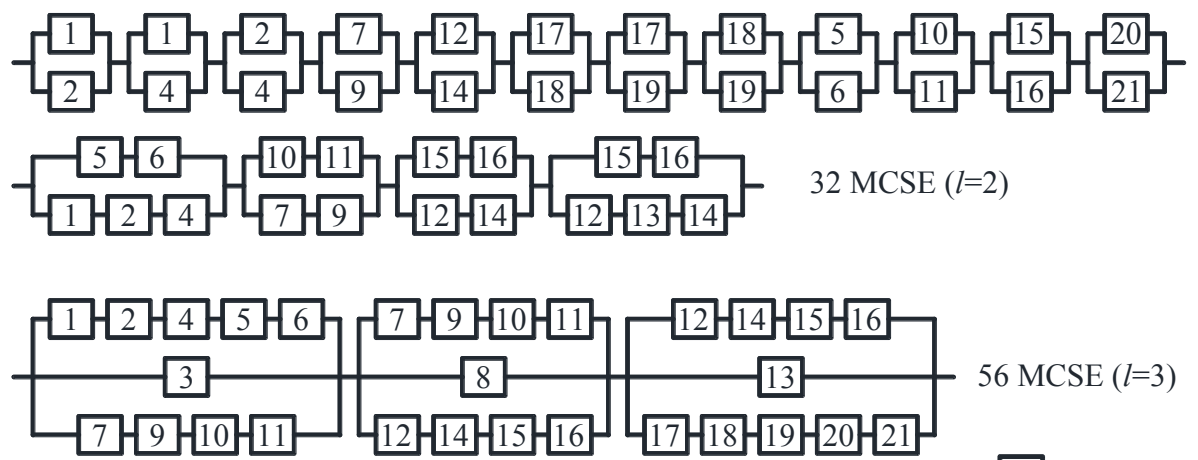

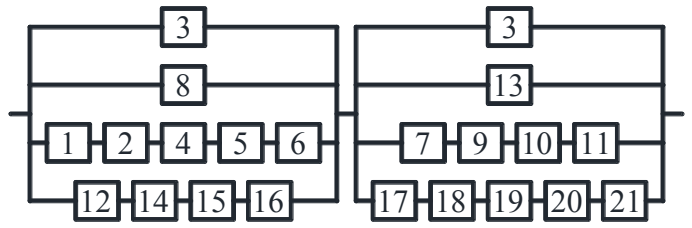

$40 \operatorname{MCSE}(l=4)$

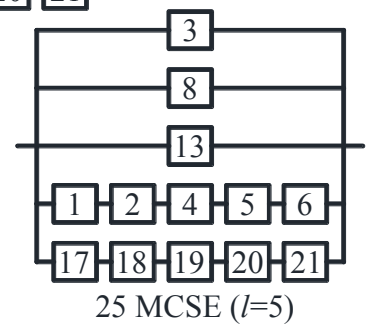

Fig. 4. Kinematically admissible failure mechanisms for the truss W1 with $n=4$ repeating sections.

When the analysis is reduced to simple formulas, it is possible to quickly evaluate the number of mechanisms in the truss structure. That is important because machine computations for the number of sections greater than four $(n>4)$ are highly time-consuming. That results from a quick increment in the number of mechanisms with an increasing number of rods, which is decisive for the duration of computations. However, formulas (6) and (7) do not reveal the elements contained in a specific mechanism. Additionally, they do not make it possible to establish the type of relation between elements, i.e. whether they are 
serial, parallel or mixed systems. Thus it is necessary to perform a full analysis. Such an analysis for the tower W1 with 4 repeating sections is presented graphically in Fig. 4.

\subsection{Reliability analysis of the truss}

In order to compare the truss towers with respect to reliability, the reliability index $\beta$ is computed for each tower of concern taking into account the most disadvantageous arrangement of loads. Reliability indexes are determined both for the assumption that all elements of the tower are connected serially, $\beta_{l}$, and considering actual connections and interactions between the rods (mixed system) taken into account. The reliability analysis considering mixed connections is performed by means of the program developed by the authors in the Mathematica environment. The reliability index $\beta_{2}$ is computed for the assumption that only parallel-serial systems are found. It is a certain simplification, allowing for a full automation of the computational process. To account for serial-parallel systems that occur in the structures, equations for individual KAFMs are worked out, and the reliability index $\beta_{3}$ is computed. It should be noted that it is a highly time-consuming process, to possibly create a serious difficulty for a larger number of elements. The results of reliability indexes for computational approaches quoted above are shown in Table 3.

Table 3. Reliability indexes of the structure.

\begin{tabular}{|c|c|c|c|c|c|}
\hline Truss topology & $l e$ & $l m$ & $\beta_{1}$ & $\beta_{2}$ & $\beta_{3}$ \\
\hline $\mathrm{W} 1$ & 21 & 153 & 1.500 & 3.454 & 3.454 \\
\hline $\mathrm{W} 2$ & 29 & 325 & 1.446 & 3.293 & 3.294 \\
\hline $\mathrm{W} 3$ & 45 & 569 & 1.320 & 2.938 & 2.938 \\
\hline
\end{tabular}

\section{Conclusions}

Truss towers, classified as multi-element pin-jointed structures, require special attention with respect to the assessment of their reliability. The analyses carried out for the study lead to some valid conclusions.

The spectral analysis of the linear stiffness matrix of truss towers makes it possible to specify the number of mechanisms and to identify parallel-serial mechanisms. On this basis, formulas for computing mechanisms for a greater number of repeating sections can be derived.

In the towers of concern for each topology, namely W1, W2, and W3, an increase, by over $100 \%$, in the reliability index is observed when the mixed system is accounted for $\left(\beta_{2}\right.$ and $\beta_{3}$ ), compared with the situation when the reliability index is computed like for the serial system $\left(\beta_{1}\right)$.

In each case the reliability index is computed, tower W1 showed the highest, whereas tower W3 - the lowest reliability value. That is related to the fact that with an increase in the number of members, the reliability of the structure decreases.

The simplification of computations proposed in the study, which consists in taking into account only the parallel-serial mechanisms in the mixed system, does not noticeably affect the reliability index of well-designed truss towers. In the case of W2 tower, the difference between $\beta_{2}$ and $\beta_{3}$ is 0.001 . Instead, this approach allows full automation of the computational process. Also, it is possible to avoid the necessity of working out all the equations for individual KAFMs found in the system. 


\section{References}

1. K. Rykaluk, Steel structures. Chimneys, towers, masts (in Polish), Oficyna Wydawnicza Politechniki Wrocławskiej, (Wrocław 2005)

2. PN-EN 1990:2004 Eurocode: Basis of structural design.

3. S. Woliński, K. Wróbel, Reliability of building structures (in Polish), Oficyna Politechniki Rzeszowskiej, (Rzeszów 2001)

4. O. Ditlevsen, H. O. Madsen, Structural Reliability Methods, Department of Mechanical Engineering Technical University of Denmark, June-September (2007)

5. A. Biegus, Probabilistic analysis of steel structures (in Polish), Wydawnictwo Naukowe PWN, (Warszawa-Wrocław 1999)

6. J. Kłosowska, P. Obara, J. Turant, IOP Conference Series: Materials Science and Engineering, Kinematically Admissible Failure Mechanisms for Plane Trusses, 245 (2017), doi:10.1088/1757-899X/245/2/022022

7. K. Kubicka, U. Radoń, Archives of Civil Engineering, Proposal for the assessment of steel truss reliability under fire conditions, 61, 4, 141-154 (2015)

8. P. Thoft-Christensen, M. Baker, Structural reliability theory and its applications, Springer-Verlag (1982)

9. PN-EN 1991-1-4 Eurocode:2008 1. Actions on structures. General actions. Wind actions.

10. PN-EN 1993-1-1 Eurocode:2006 3. Design of steel structures. Part 1-1: General rules and rules for buildings.

11. PN-EN 1993-3-1. Eurocode:2006 3. Design of steel structures. Part 3-1: Towers, masts and chimneys - Towers and masts.

12. K. Rykaluk, Inżynieria i Budownictwo, Wind load on steel towers according to Polish and European standards (in Polish), 65, 7, 367-374 (2009) 